\title{
An Integrated Approach for Displacement Prediction of Landslides Based on the Data Mining and VMD- FOA-BPNN Model
}

\section{Fasheng Miao}

China University of Geosciences

\section{Xiaoxu Xie}

China University of Geosciences

Yiping Wu ( $\nabla$ ypwu1971@163.com )

China University of Geosciences https://orcid.org/0000-0002-0536-1505

\section{Linwei Li}

China University of Geosciences

\section{Weiwei Zhan}

Clemson University

\section{Research Article}

Keywords: Baishuihe Landslide, displacement prediction, Three Gorges Reservoir, Data mining, VMD-FOABPNN

Posted Date: July 9th, 2021

DOl: https://doi.org/10.21203/rs.3.rs-661891/v1

License: (1) This work is licensed under a Creative Commons Attribution 4.0 International License. 
Fasheng Miao ${ }^{1}$

1. Faculty of Engineering, China University of Geosciences, Wuhan, China, 430074

First author. Tel.: +86 15527160870, Email: fsmiao@ cug.edu.cn

$7 \quad$ Xiaoxu $\mathbf{X i e}^{1}$

Email: xiexx@cug.edu.cn

*Corresponding author. Tel.: +86 13971170757, E-mail: ypwu1971@163.com

\section{Linwei $\mathbf{L i}^{\mathbf{1}}$}

14 1. Faculty of Engineering, China University of Geosciences, Wuhan, China, 430074

15 Email: linweili@cug.edu.cn

\section{Weiwei Zhan ${ }^{2}$}

2. Glenn Department of Civil Engineering, Clemson University, 29630

Email: wzhan@clemson.edu

\section{Acknowledgments:}

This research was supported by the National Natural Science Foundation of China (42007267, 41977244), the Fundamental Research Funds for the Central University, China University of Geosciences (Wuhan), and the National Key R\&D Program of China (2017YFC-1501301). The authors thank the colleagues in our laboratory for their constructive comments and assistance. 


\title{
An Integrated Approach for Displacement Prediction of Landslides Based
}

\section{on the Data Mining and VMD-FOA-BPNN Model}

\author{
Fasheng Miao ${ }^{1}$, Xiaoxu Xie ${ }^{1}$, Yiping $\mathrm{Wu}^{1,}{ }^{\text {* }}$, Linwei $\mathrm{Li}^{1}$, Weiwei Zhan ${ }^{2}$ \\ (1. Faculty of Engineering, China University of Geosciences, WuHan, 430074, China \\ 2. Glenn Department of Civil Engineering, Clemson University, 29630, USA)
}

\begin{abstract}
Landslide prediction is important for mitigating geohazards but is very challenging. The fluctuation of reservoir water level and rainfall are the main external triggering factors controlling the deformation of riverine landslides. In this paper, the Baishuihe landslide in the Three Gorges Reservoir area, which has a typical "step-like" behavior, is taken as the study case, and an integrated approach for landslide displacement prediction combining data mining and Variational Mode Decomposition, Fruit Fly Optimization Algorithm, Back Propagation Neural Network (VMD-FOA-BPNN) is proposed. Nine triggering factors including the reservoir level and rainfall are extracted. First, triggering factors and monthly velocity of the landslide are clustered into several categories by Two-step Clustering (TSC). Then, Apriori algorithm is used to mine the association rules between triggering factors and monthly velocity, and comprehensive contribution of each factor is calculated based on the data mining results. Next, the displacement of monitoring point ZG93 and triggering factors are decomposed by VMD based on the time series analysis of the landslide. Last, the trend term displacement is trained and predicted by the subsection functions, and FOA-BPNN models are used to train and predict the periodic and random term. The prediction results show that, compared with the current popular prediction models, the proposed model can effectively improve the prediction accuracy, which has high practicability and application value in the study of landslide displacement prediction.
\end{abstract}

Keywords: Baishuihe Landslide; displacement prediction; Three Gorges Reservoir; Data mining; VMD-FOA-BPNN

\section{Introduction}

Landslide prediction is important for mitigating geohazards but is very challenging, especially for the mountain and reservoir area. Landslides are one of the worst types of natural disasters, which occur frequently around the world (Hong et al. 2016; Juang et al. 2019). The Three Gorges Reservoir area is in the middle and upper reaches of the Yangtze River. Since the impoundment of water began in 2003, the reservoir bank has experienced periodic fluctuation of reservoir water level, which causes the rock and soil of the slope at the reservoir bank to undergo a change of dynamic osmotic pressure repeatedly. This process has a great impact on the surrounding regional geological environment, resulting in the deformation and destruction of the original stable reservoir bank, and leading to the reactivation and deformation of many ancient landslides (Tang et al., 2019). The political, economic, and social impact of a large hydropower hub is significant (Wu et al., 2017; Li et al., 2019). Therefore, it is important to carry out the research on the riverbank landslides in the Three Gorges Reservoir area (Miao et al., 2018a).

The study of landslide displacement prediction is a hot topic at the cutting edge in engineering geology (Intrieri et al., 2019). Due to the combined effect of periodic fluctuation of water level and rainfall in the Three Gorges Reservoir area, the monitoring curve of landslide displacement in the reservoir area appears "step-like" (Lenti et al., 2012), so the accuracy of a direct prediction method for the total displacement is not good enough. Therefore, the decomposition technology should be used to divide the landslide displacement into several items. At present, two main methods are used to decompose landslide displacement: (1) combining time series analysis 
method and simple moving average method, the trend displacement and periodic displacement are separated from the original displacement time series successively (Miao et al., 2018b; Yang et al., 2019). This method is simple and practical, and the physical meaning of the obtained displacement component is clear. But due to the defects of the decomposition method itself, it is unable to obtain the random displacement proposed in the time series analysis, so the approximate treatment is generally adopted in practical application. (2) using empirical mode decomposition (EMD), ensemble empirical mode decomposition (EEMD) or wavelet analysis to decompose the displacement (Lian et al., 2014; Huang et al., 2017; Shihabudheen et al., 2017). Combined with the time series of landslide displacement, these methods can make clear the physical meaning of each part of displacement and reflect the relationship between each displacement component and the triggering factors to improve the accuracy of displacement prediction.

From the initial empirical model to the mathematical statistical model, and then to the nonlinear theoretical model and comprehensive model, the landslide prediction model has experienced rapid development in the past 50 years. Nowadays, with the development of high-speed computers, various machine learning models have been widely used in landslide displacement prediction, and many breakthroughs have been achieved. At present, the commonly used models for the displacement prediction include Back Propagation Neural Network (BPNN) (Chen and Zeng, 2013; Fu et al., 2021; Zhang et al., 2021), Support Vector Regression (SVR) (Liu et al., 2020; Dong et al., 2021), Extreme Learning Machine (ELM) (Lian et al., 2014), Evaluating Machine Learning (EML) (Goetz et al., 2015), Kernel Extreme Learning Machine (KELM) (Zhou et al., 2018; Li et al., 2021), Long Short-Term Memory (LSTM) (Xu et al., 2018; Yang et al., 2019), and so on. And many algorithms are used to optimize parameters for the prediction models, including Genetic Algorithm (GA) (Li and Kong, 2014), Grid Search algorithm (GS) (Miao et al., 2018b), Particle Swarm Optimization (PSO) (Zhou et al., 2016), Grey Wolf Optimizer (GWO) (Guo et al., 2019), Fruit Fly Optimization Algorithm (FOA) (Wang et al., 2019), and so on. In the development of landslide displacement prediction model, the selection of influencing factors is very important. For the landslides in the reservoir area, the fluctuation of reservoir level and rainfall are usually used as the hydrologic triggering factors of landslide deformation and failure (Hong et al., 2017). In the landslide prediction model, the increase of input factors does not necessarily lead to higher prediction accuracy. For different types of displacement, it is necessary to select the appropriate inducing factor as the input layer to establish the model. In order to optimize the triggering factors to find the most suitable factors for displacement prediction, data mining technology could be used.

In this paper, the Baishuihe landslide in the Three Gorges Reservoir area was taken as an example, and an integrated approach for landslide displacement prediction combining data mining and VMD-FOA-BPNN was proposed. A total of ten triggering factors were extracted. Two-step Clustering (TSC) and Apriori algorithm were used to mine the association rules and comprehensive contribution of each factor. Based on the time series analysis of landslides, the displacement of monitoring point ZG93 and triggering factors are decomposed by VMD. The trend term displacement was trained and predicted by one-dimensional cubic subsection functions, and FOA-BPNN models were used to train and predict the periodic and random terms. A flow chart is shown in Fig. 1. 


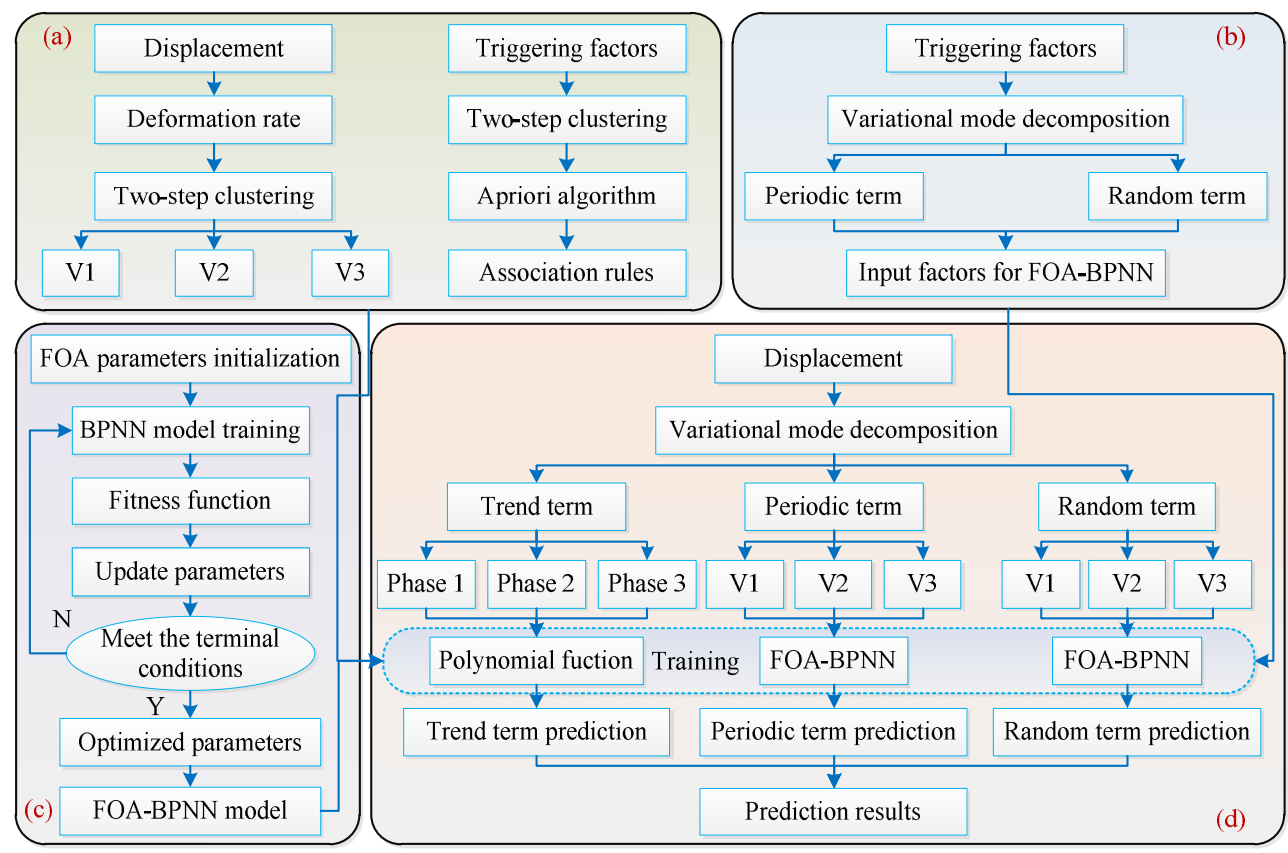

Fig. 1 Flow chart of the displacement prediction (a) data mining process; (b) decomposition of triggering factors; (c) optimization procedure of FOA-BPNN; (d) displacement prediction

\section{Methodology}

\subsection{Apriori algorithm}

Apriori algorithm was first proposed by Agrawal and Srikant and has become the core algorithm of association rule mining (Agrawal et al., 1994). This algorithm can deal only with categorical variables rather than numeric variables. The algorithm mainly includes two steps: (1) generate frequent item sets that meet the minimum support; (2) association rules that satisfy the minimum credibility are generated in the frequent item set generated in the first step (Perego et al., 2001).

Frequent item set $T$ contains item $a$. If its support is equal to or greater than the support threshold specified by the user, that is:

$$
\frac{|T(a)|}{|T|} \geqslant \text { minsupp }
$$

Then $a$ is called frequent item set. The set including 1 item (length 1 ) is called frequent 1 item set, which is recorded as $L_{1}$. As shown in Fig. 2, $a, b, c, d$ at the bottom layer can be called frequent 1-term set when the minimum support degree is met. The frequent item set with $k$ items is called frequent $k$ item set, which is recorded as $L_{k}$. The upper-level project sets $a b, a b c$ and $a b c d$ are frequent $k$-item sets when they meet the minimum support. Apriori algorithm uses the iterative method of layer-by-layer search to generate frequent item sets. Frequent $k$-item sets are used to explore and generate $(k+1)$ item sets. The algorithm implementation process is shown in Fig. 2. 


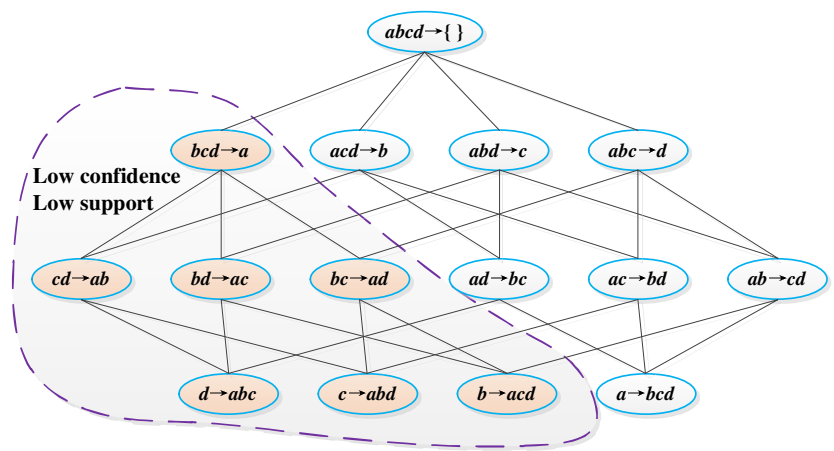

Fig. 2 The algorithm implementation process of Apriori

Simple association rules are generated from frequent item sets, and association rules with confidence greater than the threshold value are selected to form an effective rule set. For each frequent item set $L$, calculate the confidence of all non-empty subsets $L^{\prime}$. If $C_{L \rightarrow L-L^{\prime}}$ is greater than the confidence threshold specified by the user, that is:

$$
C_{L^{\prime} \rightarrow(L-L)}=\frac{|T(L)|}{\left|T\left(L^{\prime}\right)\right|} \geqslant \min \mathrm{conf}
$$

Then, the association rule $L^{\prime} \Rightarrow\left(L-L^{\prime}\right)$ can be generated.

\subsection{VMD}

VMD (Variational Mode Decomposition) was proposed by Dragomiretskiy and Zosso based on the EMD model (Dragomiretskiy and Zosso, 2013). VMD is an adaptive, completely non recursive method of modal variation and signal processing. This technology has the advantage of determining the number of modal decompositions. Its self-adaptability lies in determining the number of modal decompositions of the given sequence according to the actual situation. In the subsequent search and solution process, it can adaptively match the optimal center frequency and limited bandwidth of each mode, and can realize the effective separation of the intrinsic mode function (IMF) and the division of the signal frequency domain, and then get the effective decomposition components of the signal. Finally, optimal solutions of the variational problem can be obtained. The core idea of VMD is to construct and solve the variational problem.

For the construction of variation, assuming that the original signal $f(t)$ is decomposed into $K$ components, the corresponding constraint variation expression is:

$$
\left\{\begin{array}{l}
\min _{\left\{u_{k}\right\}\left\{\omega_{k}\right\}}\left\{\sum_{k=1}^{K}\left\|\partial_{t}\left[\left(\sigma(t)+\frac{j}{\pi t}\right) * u_{k}(t)\right] \mathrm{e}^{-j \omega_{k} t}\right\|_{2}^{2}\right\} \\
\text { s.t. } \sum_{k=1}^{K} u_{k}=f(t)
\end{array}\right\}
$$

where $\left\{u_{k}\right\}$ is the component obtained after decomposition; $\left\{\omega_{k}\right\}$ is the actual center frequency of each IMF component; $\partial_{t}$ is the Dirac function; * is the Convolution operator; $(\sigma(t)+j / \pi t) * u_{k}(t)$ is the analytical signal of each component; $e^{-j \omega_{k} t}$ is the estimated center frequency of each analytical signal.

The Lagrange multiplication operator $\lambda$ is introduced to solve Eq. (8), and the constrained variational problem is transformed into the unconstrained variational problem, as following:

$$
L\left(\left\{u_{k}\right\},\left\{\omega_{k}\right\}, \lambda\right)=\alpha \sum_{k}\left\|\partial_{t}\left[\left(\sigma(t)+\frac{j}{\pi t}\right) * u_{k}(t)\right] \mathrm{e}^{-j \omega_{k} t}\right\|_{2}^{2}+\left\|f(t)-\sum_{k} u_{k}(t)\right\|_{2}^{2}+\left\langle\lambda(t), f(t)-\sum_{k} u_{k}(t)\right\rangle
$$

where $\lambda$ is the Lagrange multiplier.

By using the alternative direction method of multipliers (ADMM), the saddle point of the above-unconstrained model can be obtained, which is the optimal solution of the constrained variational model, so that the original 
signal can be decomposed into IMF components.

VMD overcomes the problems of end-point effect and modal component overlapping associated with the EMD method, which has a more solid mathematical theoretical basis. It can reduce the non-stationary of time series with high complexity and strong nonlinearity, and decompose multiple subsequences with different frequency scales and relatively stationary, which are suitable for non-stationary sequences.

\subsection{FOA}

FOA (Fruit Fly Optimization Algorithm) is a new method of global optimization based on the foraging behavior of Drosophila melanogaster, which was proposed by Wen-Tsao Pan (Pan, 2012). The fruit fly is superior to other species in sensory perception, especially in smell and vision. The olfactory organ of Drosophila can well collect all kinds of smells floating in the air and can even smell food sources 40 kilometers away. Then, after flying to the vicinity of the food location, they can use their sharp vision to find the food or observe the gathering position of their companions, and fly in that direction.

The steps of the FOA process are as follows:

(1) Random initialization of fruit fly population position.

(2) Give individuals random distance and direction to search for food by olfaction.

(3) Since the position of food cannot be known, the distance from the origin (Dist) should be estimated first, and then the taste concentration judgment value $(S)$ is calculated, which is the reciprocal of the distance.

(4) $S$ was substituted into the taste function (or Fitness function) to get the taste concentration of the individual position.

(5) Identify the fruit fly with the highest taste concentration in these populations.

(6) The coordinates of the best taste concentration value are reserved, and then the fruit fly population flies to this position by vision.

(7) Enter the iterative optimization process. Repeat steps (2)-(5) and judge whether the taste concentration is better than that of the previous iteration. If so, carry out the step 6 .

\subsection{BPNN}

BPNN (Back Propagation Neural Network) is a kind of multilayer feedforward neural network which is trained according to the error back propagation algorithm. As the most widely used neural network, BPNN was proposed by Rumelhart and McClelland (McClelland et al., 1986). BPNN has the abilities of arbitrary complex pattern classification and excellent multi-dimensional function mapping. It solves "exclusive or" (XOR) and some other problems that simple perceptron can't solve. In terms of structure, BPNN has an input layer, a hidden layer, and an output layer. BP algorithm takes the square of network error as the objective function and uses gradient descent method to calculate the minimum value of the objective function.

The basic BPNN includes two processes: the forward propagation of signal and the back propagation of error. That is to say, the error output is calculated according to the direction from input to output, while the adjustment of weight and threshold is carried out from output to input. In the forward propagation, the input signal acts on the output node through the hidden layer and generates the output signal through a nonlinear transformation. If the actual output does not match the expected output, the error is transferred into the back-propagation process. Error retransmission occurs when the output error is retransmitted to the input layer by layer through the hidden layer, and the error is allocated to all cells of each layer, and the error signal obtained from each layer is used as the basis for adjusting the weight of each cell. By adjusting the connection strength between the input node and the hidden layer node, the connection strength between the hidden layer node and the threshold value, the error is reduced along the gradient direction. After repeated learning and training, the network parameters (weight and threshold value) corresponding to the minimum error are determined, and the training stops. At this time, the trained neural network can process the information of non-linear transformation with the minimum output error for the input 
information of similar samples.

198

199

200

201

\section{Case study: Baishuihe landslide}

\subsection{Geological conditions}

Baishuihe Landslide is in Zhigui County of the Three Gorges Reservoir area, which is approximately $56 \mathrm{~km}$ away from the dam site. The landslide is located on the South Bank (convex bank) of the Yangtze River. The elevation of the landslide gradually decreases from south to north. The elevation of the toe and rear edges is about $70 \mathrm{~m}$ and $400 \mathrm{~m}$, and the bedrock ridge is the boundary between the east and west sides. The deformation of the middle and front part of the landslide is relatively strong. The pinnate fissures are continuously distributed on both sides of the boundary, and the boundary between the east side and the rear edge is basically connected. The north-south and east-west length of the landslide are about $600 \mathrm{~m}$ and $700 \mathrm{~m}$, respectively. The Baishuihe Landslide formed in a nearly north-south gully sloping to the north and spread into the Yangtze River. The slope of the landslide is $30^{\circ}$ to $35^{\circ}$, and an average thickness of $30 \mathrm{~m}$ with a volume of $1.26 \times 10^{7} \mathrm{~m}^{3}$. The topographic map and schematic geological profile of the Baishuihe Landslide is shown in Fig. 3 and 4.

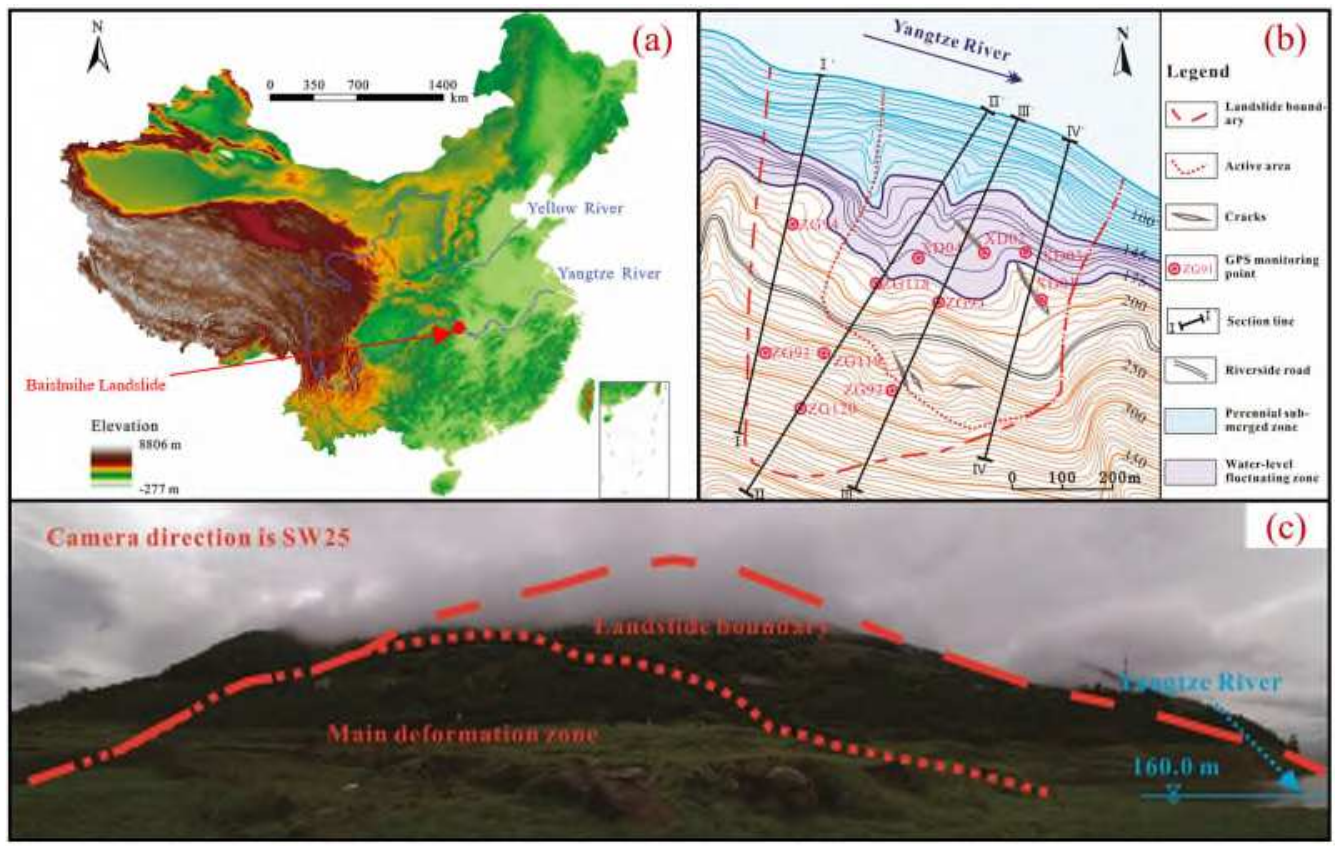

Fig. 3 (a) Location of the Baishuihe Landslide; (b) Topographic map of the Baishuihe Landslide; (c) Overall view of the Baishuihe Landslide

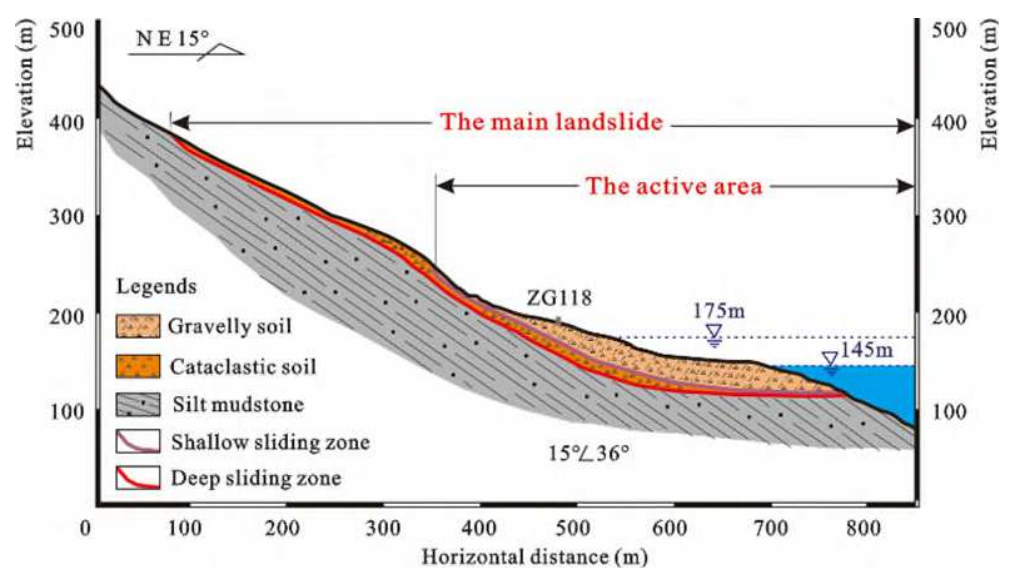

Fig. 4 Schematic Geological Profile of the Baishuihe Landslide (II-II') 


\subsection{Deformation of the landslide}

The Baishuihe Landslide has been monitored since the reservoir water level reached $135 \mathrm{~m}$ in June 2003, and the monitoring surface layout is shown in Fig. 3(b). According to the characteristics of surface monitoring displacement and surface macro deformation, Baishuihe Landslide was divided into two areas: (1) the active area (area $A$ ) is the middle and front part of the landslide with strong deformation. After the completion of the Three Gorges Dam, the landslide has produced obvious displacement due to the impoundment of the reservoir. Several transverse tension cracks appear in the east of the landslide. The eastern and posterior boundaries are basically connected, and the western boundary cracks are in the shape of cracks pinnately distributed. From August 2005 to August 2006, there were many landslides on the inner side slope of the riverside highway with the landslide elevation of about $220 \mathrm{~m}$, and many subsidence and tension cracks appeared on the surface of the landslide. From June 2007 to September 2007, approximately $100,000 \mathrm{~m}^{3}$ of landslide piled on the road in the rear of the active area (Fig. 5). From then on to August 2009, the landslide displacement continued to "step" development. (2) The relatively stable area $(\operatorname{area} B$ ) is the middle and rear of the landslide, where the accumulated deformation is small, and the movement rate is slow, only $1.5 \sim 4.0 \mathrm{~mm} / \mathrm{a}$.

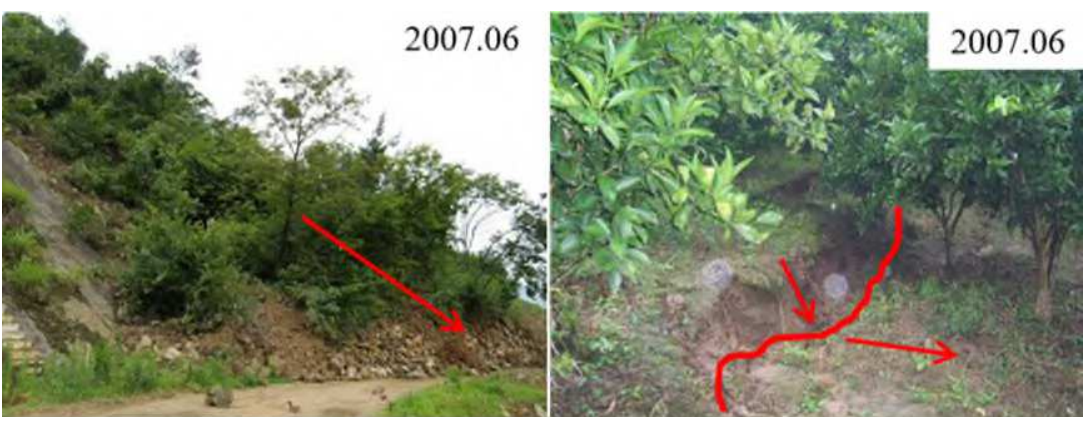

Fig. 5 Macroscopic deformation of the Baishuihe Landslide

\subsection{Analysis of the monitoring data}

There are 3 monitoring sections and 6 GPS monitoring points in the Baishuihe Landslide active area. Among them, the monitoring points ZG93 and ZG118 were started in June 2003, and XD01, XD02 and XD03, XD04 were added in May and October 2005 respectively. It can be observed that the displacement of each monitoring point was synchronous. The monitoring period of ZG93, ZG118 and XD01 was long, which was representative and can reflect the whole movement process of the landslide. Therefore, in this paper, these three points were taken for detailed analysis (Fig. 6). According to the filling scheduling of the reservoir, the monitoring data can be divided into 3 stages for analysis:

(1) Phase I (from June 2003 to June 2006): The water level of the reservoir started from $135 \mathrm{~m}$ in September and reached the highest level of $139 \mathrm{~m}$ in October. The maximum displacement of ZG93 and ZG118 was $25.8 \mathrm{~mm}$ and $30.6 \mathrm{~mm}$ respectively during the three impoundment periods. The reservoir basically maintains the highest water level from November to January of the next year, and the maximum monthly displacement rates of these three points were under $13 \mathrm{~mm} /$ month respectively, which was relatively slow. The water level began to drop in February every year and reached the lowest level $(135 \mathrm{~m})$ in July. During this period, the minimum increase of these three points was over $80 \mathrm{~mm}$, and the maximum increase was over $150 \mathrm{~mm}$. Especially in May and June, the increase rate of landslide displacement was the largest. From the end of July to the beginning of September, the reservoir water level maintained the lowest level, but the landslide displacement first continued to grow rapidly and then basically remained the same. It can be observed that a heavy rainfall in July 2005 did not increase 
displacement sharply.

(2) Phase II (from July 2006 to June 2008): In this phase, the reservoir water level fluctuated between 145 and $155 \mathrm{~m}$. During April to June 2007, the reservoir level dropped from $155 \mathrm{~m}$ to $145 \mathrm{~m}$ for the first time. This large drop in the reservoir water level led to the increase of the hydrodynamic pressure in the landslide, and changed the seepage field of the landslide, which resulted in the first sudden increase in the displacement of each monitoring point appear. The displacement increased more than $1000 \mathrm{~mm}$.

(3) Phase III (from July 2008 to December 2016): The reservoir water level fluctuated between 145 and $175 \mathrm{~m}$. Before 2015, the annual displacement rate showed a downward trend. The displacement of XD01 on the right side of landslide was obviously larger than that of other monitoring points. The deformation on the right side of the landslide was large, which was consistent with the field investigation results. The cracks of Baishuihe Landslide were mainly concentrated near the right edge of the landslide. In June 2015, the reservoir water level rose rapidly after a short period of rapid decline due to the impact of the "Oriental Star" cruise ship accident. The reservoir water level dropped rapidly from $153.34 \mathrm{~m}$ to $145.22 \mathrm{~m}$ with a daily average decline of $0.68 \mathrm{~m} / \mathrm{d}$ (Fig. 6). During the same time, several successive heavy rainfalls occurred in Shazhenxi town. Total precipitation was about 265.9 $\mathrm{mm}$, accounting for $36 \%$ of the total rainfall in the flood period of that year. Under the combined effect of continuous heavy rainfall and rapid decline of reservoir water level, large-scale deformation of the landslide occurred.

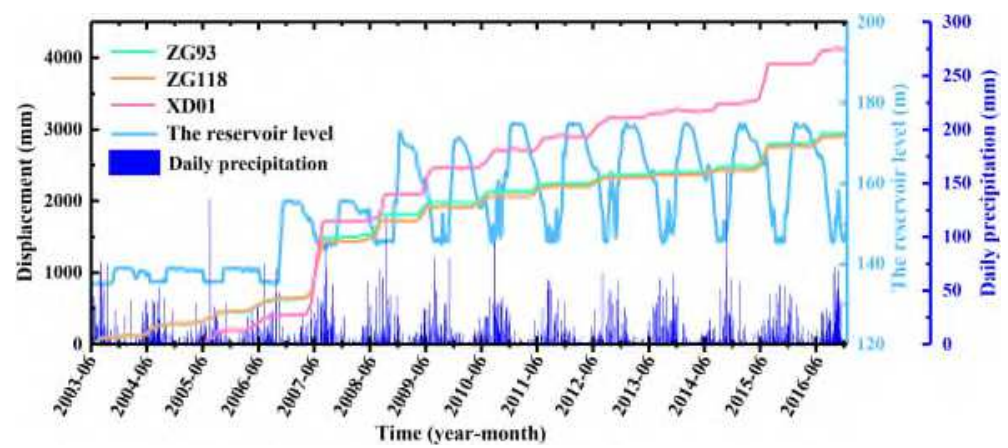

Fig. 6 Long term monitoring data of the Baishuihe Landslide (displacement, reservoir level, precipitation)

\section{Results}

\subsection{Triggering factors}

The fluctuation of reservoir level and rainfall are usually the main triggering factors affecting the deformation of the reservoir bank landslide. In this paper, ZG93 monitoring data was selected for the following reasons: (1) ZG93 was the earliest deformation monitoring point with complete monitoring period; (2) ZG93 monitoring point was in the middle of the landslide, which can basically reflect the deformation trend of the landslide. Monitoring points ZG93, ZG118 and XD01 have similar deformation characteristics. Therefore, monitoring data of points ZG118 and XD01 were added to increase the sample size and overcome model overfitting error, as well as to provide a better representative prediction of the overall landslide displacement. In this research, a total of ten triggering factors were selected to carry out displacement prediction research, including five reservoir level related factors, four rainfall related factors, and one deformation factor, as shown in Table 1. Among them, nine factors related to reservoir water level and rainfall were external triggering factors, while the deformation rate of the last month (F10) was an internal factor, which could reflect the deformation and failure of the landslide itself.

Table 1 Triggering factors to carry out displacement prediction

\begin{tabular}{lll}
\hline No. & Factors & Category \\
\hline
\end{tabular}




\begin{tabular}{ccc}
\hline F1 & Monthly average water level $(\bar{h})(\mathrm{m})$ & Reservoir water level \\
F2 & Monthly maximum daily drop of water level $\left(\Delta h_{\max }^{\text {daily drop }}\right)(\mathrm{m} /$ day $)$ & Reservoir water level \\
F3 & Monthly maximum daily rise of water level $\left(\Delta h_{\mathrm{max}}^{\text {daily }}\right.$ rise $)(\mathrm{m} /$ day $)$ & Reservoir water level \\
F4 & Monthly fluctuation of water level $\left(\Delta h^{\text {month }}\right)(\mathrm{m} / \mathrm{month})$ & Reservoir water level \\
F5 & Bimonthly fluctuation of water level $\left(\Delta h^{2 \text { month }}\right)(\mathrm{m} / 2-\mathrm{month})$ & Reservoir water level \\
F6 & Monthly maximum effective continuous rainfall $\left(q_{\text {continuous }}^{\text {effective }}\right)(\mathrm{mm})$ & Rainfall \\
F7 & Monthly cumulative rainfall $\left(q^{\text {month }}\right)(\mathrm{mm})$ & Rainfall \\
F8 & Bimonthly cumulative rainfall $\left(q^{\text {2month }}\right)(\mathrm{mm})$ & Rainfall \\
F9 & Monthly maximum daily rainfall $\left(q_{\max }^{\text {day }}\right)(\mathrm{mm})$ & Rainfall \\
F10 & Monthly velocity $(v)(\mathrm{mm} / \mathrm{month})$ & Deformation \\
\hline
\end{tabular}

288

\subsection{Clustering results}

Based on the TSC (Two-step Clustering) algorithm, the induced factors were clustered. Maximum and minimum categories of each triggering factors were set as 10 and 2 respectively. The distance measurement method used in the two-step clustering algorithm was Euclidean distance, and the cluster criterion are the Bayesian Information Criterion (BIC). Clustering results of the external triggering factors are shown in Tables 2 and 3 . Monthly velocity ( $v$ ) was clustered into three categories (Low V1; Medium V2; High V3), as shown in Table 4.

Table 2 Clustering results of the reservoir water level factors (ZG93, ZG118, XD01)

\begin{tabular}{|c|c|c|c|c|}
\hline No. & Factors & \multicolumn{2}{|c|}{ Clustering results } & Coun \\
\hline \multirow{3}{*}{$\mathrm{F} 1$} & \multirow{3}{*}{$\bar{h}$} & $(135.13 \sim 138.95)$ & High-Water-Level (F11) & 97 \\
\hline & & $(144.21 \sim 158.02)$ & Medium-Water-Level (F12) & 186 \\
\hline & & $(160.14 \sim 174.74)$ & Low-Water-Level (F13) & 183 \\
\hline \multirow{3}{*}{$\mathrm{F} 2$} & \multirow{3}{*}{$\Delta h_{\max }^{\text {daily drop }}$} & $(-0.14 \sim 0.58)$ & Slow-Daily-Drop (F21) & 339 \\
\hline & & $(0.63 \sim 1.87)$ & Medium-Daily-Drop (F22) & 92 \\
\hline & & $(1.91 \sim 3.69)$ & Sharp-Daily-Drop (F23) & 35 \\
\hline \multirow{2}{*}{ F3 } & \multirow{2}{*}{$\Delta h_{\text {max }}^{\text {daily rise }}$} & $(-0.43 \sim 0.04)$ & Slow-Daily-Rise (F31) & 129 \\
\hline & & $(-1.70 \sim-0.49)$ & Sharp-Daily-Rise (F32) & 337 \\
\hline \multirow{2}{*}{ F4 } & \multirow{2}{*}{$\Delta h^{\text {month }}$} & $(0 \sim 6.18)$ & Smooth-Fluctuation (F41) & 349 \\
\hline & & $(6.59 \sim 18.25)$ & Sharp-Fluctuation (F42) & 117 \\
\hline \multirow{3}{*}{ F5 } & \multirow{3}{*}{$\Delta h^{2 m o n t h}$} & $(0 \sim 6.50)$ & Non-Fluctuation (F51) & 250 \\
\hline & & $(6.68 \sim 14.15)$ & Smooth-Fluctuation (F52) & 126 \\
\hline & & $(14.91 \sim 28.71)$ & Sharp-Fluctuation (F53) & 90 \\
\hline
\end{tabular}

Table 3 Clustering results of the rainfall factors (ZG93, ZG118, XD01)

\begin{tabular}{ccccc}
\hline No. & Factors & & Clustering results & Count \\
\hline & & $(1.50 \sim 30.30)$ & Light-Effective-Rainfall (F61) & 182 \\
\multirow{2}{*}{ F6 } & $q_{\text {continuous }}^{\text {effectiv }}$ & $(31.30 \sim 66.00)$ & Moderate-Effective-Rainfall (F62) & 151 \\
& & $(67.70 \sim 110.50)$ & Middle-Effective-Rainfall (F63) & 92 \\
& & $(125.00 \sim 239.40)$ & Heavy-Effective-Rainfall (F64) & 41 \\
\hline \multirow{3}{*}{ F7 } & \multirow{2}{*}{$q^{\text {month }}$} & $(3.10 \sim 66.10)$ & Light-Effective-Rainfall (F71) & 198 \\
& & $(69.90 \sim 163.70)$ & Moderate-Effective-Rainfall (F72) & 191 \\
& & & & \\
\hline
\end{tabular}




\begin{tabular}{|c|c|c|c|c|}
\hline & & $(357.50 \sim 517.60)$ & Heavy-Effective-Rainfall (F74) & 17 \\
\hline \multirow{3}{*}{ F8 } & \multirow{3}{*}{$q^{2 m o n t h}$} & $(18.40 \sim 135.20)$ & Light-Effective-Rainfall (F81) & 197 \\
\hline & & $(143.60 \sim 362.90)$ & Moderate-Effective-Rainfall (F82) & 212 \\
\hline & & $(367.20 \sim 726.30)$ & Heavy-Effective-Rainfall (F83) & 57 \\
\hline \multirow{4}{*}{ F9 } & \multirow{4}{*}{$q_{\max }^{d a y}$} & $(1.30 \sim 25.60)$ & Light -Daily-Rainfall (F91) & 234 \\
\hline & & $(26.50 \sim 51.30)$ & Moderate-Daily-Rainfall (F92) & 151 \\
\hline & & $(51.70 \sim 95.80)$ & Middle-Daily-Rainfall (F93) & 72 \\
\hline & & $(121.10 \sim 160.70)$ & Heavy-Daily-Rainfall (F94) & 9 \\
\hline
\end{tabular}

299

300

301

Table 4 Clustering results of the monthly velocity (ZG93, ZG118, XD01)

\begin{tabular}{ccc}
\hline Monthly velocity $(v)(\mathrm{mm} /$ month) & Clustering results & Count \\
\hline$(-9.61 \sim 21.66)$ & Low (V1) & 358 \\
$(22.35 \sim 81.89)$ & Medium (V2) & 81 \\
$(137.70 \sim 313.24)$ & High (V3) & 27 \\
\hline
\end{tabular}

\subsection{Data mining and association rules}

In the data mining process, nine triggering factors of the landslide $\left(\bar{h}, \Delta h_{\text {max }}^{\text {daily drop }}, \Delta h_{\text {max }}^{\text {daily rise }}, \Delta h^{\text {month }}\right.$, $\left.\Delta h^{2 m o n t h}, q_{\text {continuous }}^{\text {effective }}, q^{\text {month }}, q^{2 m o n t h}, q_{\max }^{\text {day }}\right)$ were set as the former item of association rules, and the deformation rate (Monthly velocity $v$ ) was set as the consequent item. In the Apriori algorithm, the minimum conditional support was set to 0.01 , and the minimum rule confidence was set to $100 \%$ (ensure that the mining association criteria were absolutely correct). A total of 5447 association rules were generated, most of which were V1 and V2 stages of the landslide (4247 and 1008 respectively). The main factors controlling V1 deformation of the landslide were smooth fluctuation of reservoir level and weak rainfall. The main factors controlling the V2 deformation of the landslide were the sharp fluctuation of reservoir water level and medium heavy rainfall. And the main factor controlling the V3 deformation of the landslide was heavy rainfall. According to the Baishuihe landslide monitoring data, rainfall was generally concentrated in June to September every year, and the reservoir was controlled from high water level to low water level before this period. In other words, there was a certain negative correlation between monthly rainfall and monthly average water level elevation. This period was also the time when the landslide deformation was severe.

Statistics results of the data mining and association rules are shown in Table 5. Total support, average support, and contribution without support of each triggering factor were counted, and the comprehensive contribution was the mean value of these three contributions. Comprehensive contribution of each factor according to the association rules is shown in Fig. 7. Factors with contribution degree less than 0.3 were eliminated and were not used as input layer in the prediction model. Therefore, eight triggering factors were taken as the input layer in the V1 and V3 prediction models (F1, F3, F5, F6, F7, F8, F9, F10), and eight triggering factors were taken as the input layer in the V2 prediction model (F1, F2, F5, F6, F7, F8, F9, F10).

Table 5 Statistics results of the data mining and association rules

\begin{tabular}{cccccccccccc}
\hline & Contribution & F1 & F2 & F3 & F4 & F5 & F6 & F7 & F8 & F9 \\
\hline \multirow{4}{*}{ V1 } & Association rules & 2860 & 1936 & 2071 & 1683 & 2673 & 2780 & 2610 & 2770 & 2630 \\
& Total support & 4480.98 & 1867.49 & 3231.69 & 1557.06 & 3723.31 & 3776.06 & 3579.76 & 3800.01 & 3744.18 \\
& Average support & 1.57 & 0.96 & 1.56 & 0.93 & 1.39 & 1.36 & 1.37 & 1.37 & 1.42 \\
& Contribution without support & 0.67 & 0.45 & 0.49 & 0.40 & 0.63 & 0.65 & 0.61 & 0.65 & 0.62 \\
\hline
\end{tabular}




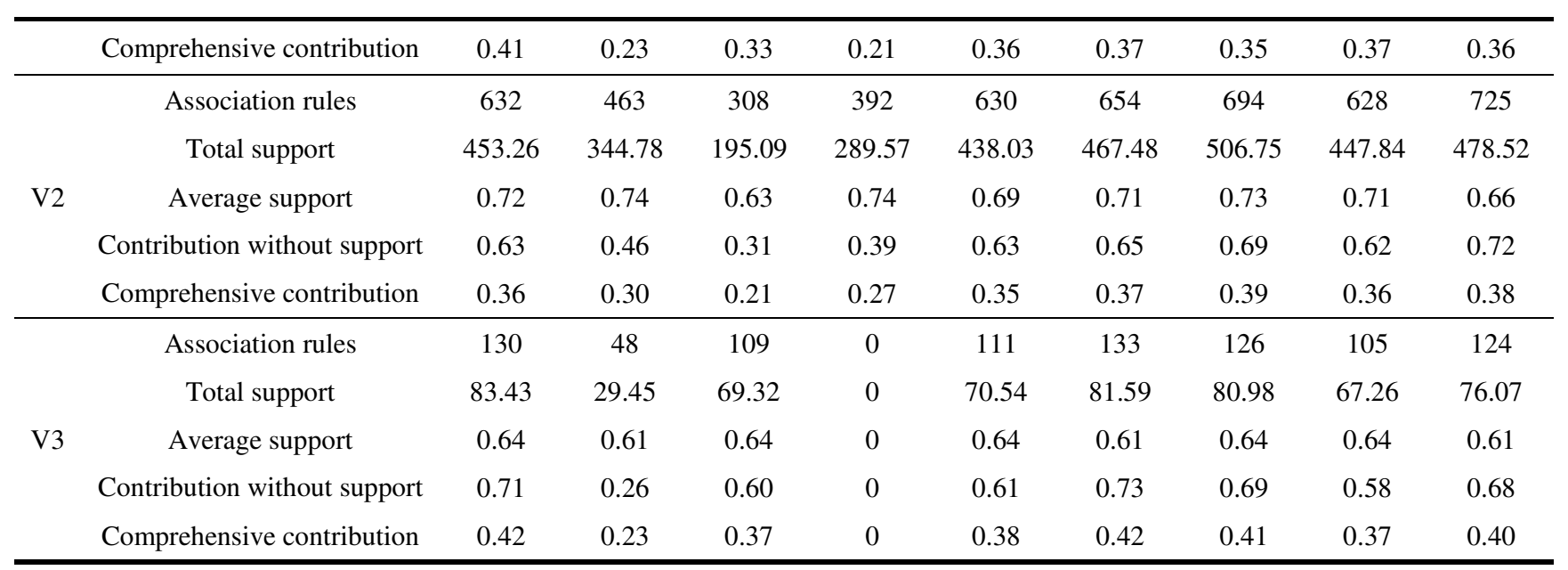

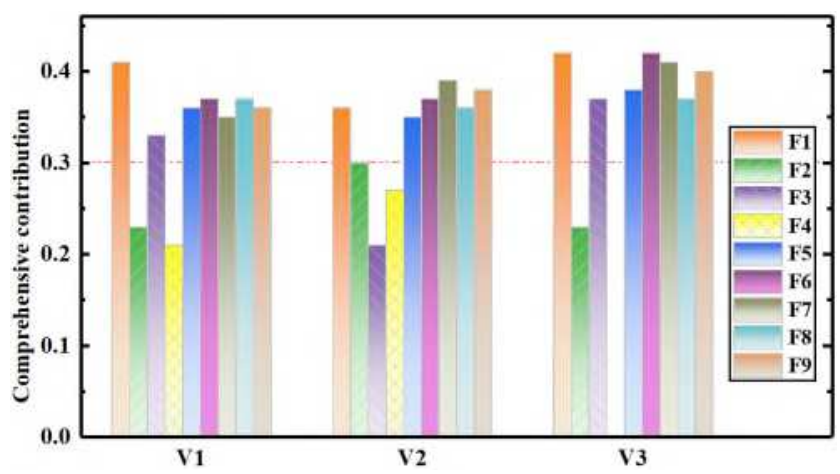

Fig. 7 Comprehensive contribution of each factor according to the association rules

\subsection{VMD decomposition}

Before using VMD to decompose the displacement of ZG93, it is necessary to know the modal number $K$ in the object to be decomposed in advance. In order to ensure that each component obtained by decomposition has practical physical significance and reduce the possibility of false components, according to the time series theory of landslide deformation, landslide displacement can be divided into three parts:

$$
X(t)=\alpha(t)+\beta(t)+\gamma(t)
$$

Where $X(t)$ is the observed value of landslide displacement, $\alpha(t)$ is the trend displacement, $\beta(t)$ is the periodic displacement and $\gamma(t)$ is the random displacement.

Therefore, $K=3$ was determined when the landslide displacement was decomposed. At the same time, in order to ensure the fidelity of the displacement time series after decomposition, aiming at the decomposition effect (residual term) of displacement decomposition, the penalty parameter $a$ and the rising step $\tau$ ( $a=1.5$ and $\tau=0.1$ ) were finally determined through multiple trials. Using the above parameter settings to decompose the displacement data, the decomposed high-frequency component corresponds to the random displacement, the low-frequency component corresponds to the periodic displacement, and the residual component corresponds to the trend displacement. Composition of training and prediction samples are shown in Table 6.

When decomposing the triggering factors, $K$ was set to 2 . In order to ensure the time sequence of these factors after decomposing, in this paper, decomposition effect of low-frequency factors was set as the object, and the penalty parameter $a$ and the rising step $\tau$ were finally determined through multiple trials $(a=700, \tau=0.5)$, as shown in Fig. 8. 


\begin{tabular}{ccccccc}
\hline Samples & \multicolumn{3}{c}{ Training samples } & \multicolumn{3}{c}{ Prediction samples } \\
\hline Monthly velocity & V1 & V2 & V3 & V1 & V2 & V3 \\
ZG93 & 116 & 30 & 5 & 10 & 2 & 0 \\
ZG118 & 119 & 24 & 8 & 10 & 2 & 0 \\
XD01 & 93 & 22 & 13 & 10 & 1 & 1 \\
Total samples & 328 & 76 & 26 & 30 & 5 & 1 \\
\hline
\end{tabular}

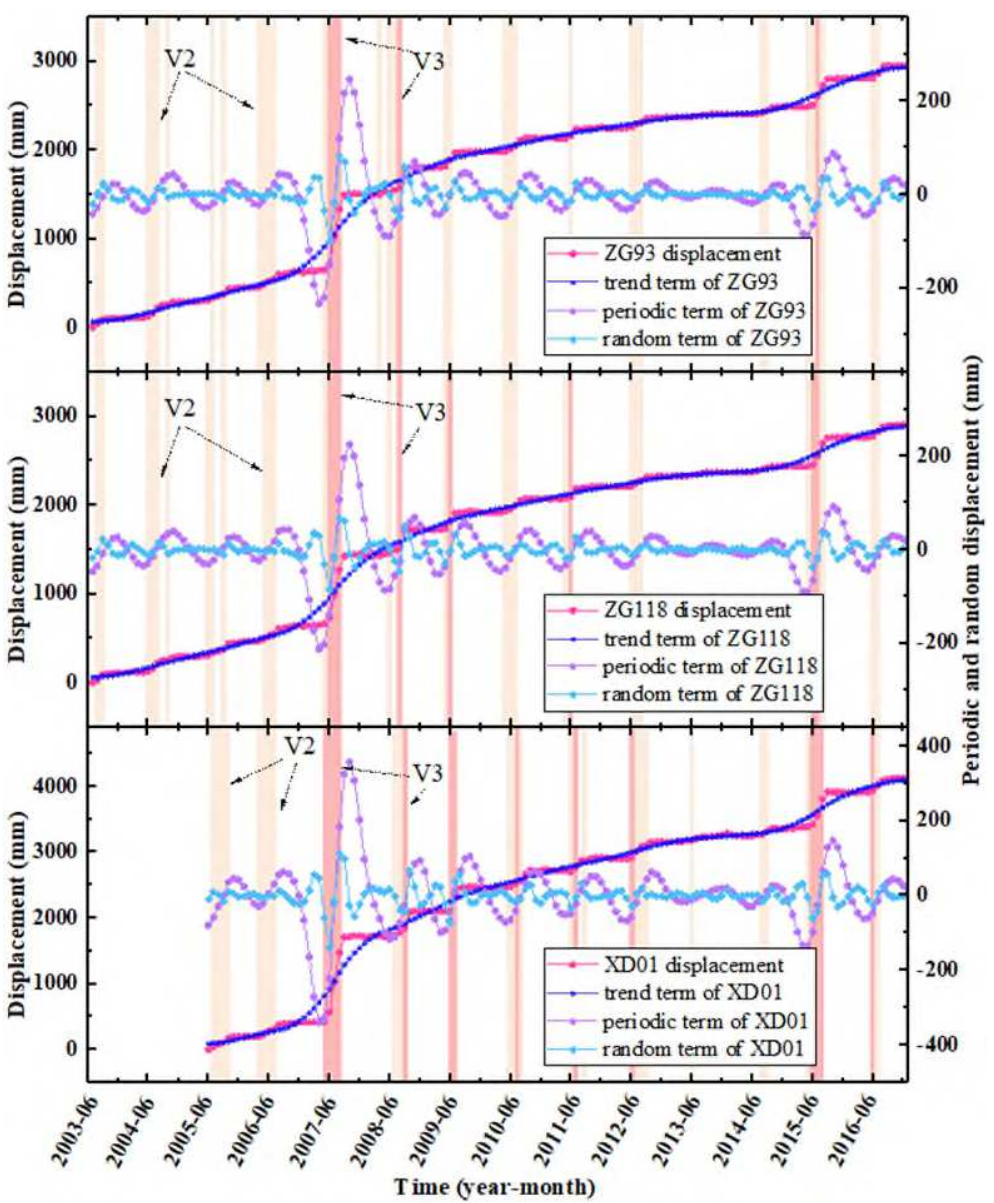

Fig. 8 VMD decomposition of monitoring displacements (ZG93, ZG118, XD01)

\subsection{Displacement prediction}

\section{Trend term prediction}

The displacement of the trend term showed a distinct piecewise function. Therefore, the trend term of ZG93 was divided into three phases: phase1 (2003.06 2007.06), phase 2 (2007.06 2014.06), and phase 3 (2014.06 2016.12). Based on the robust least square method, the following fitting is used to find the functional relationship between the trend displacement and time of the monitoring point:

$$
S=a t^{3}+b t^{2}+c t+d
$$

The fitting and prediction curves of the trend term are shown in Fig. 9, and the parameters of the fitting functions are shown in Table 7. It can be known that the prediction accuracy $\mathrm{R}^{2}$ of the trend term was over $99 \%$, and the RMSE was only 4.063 . 


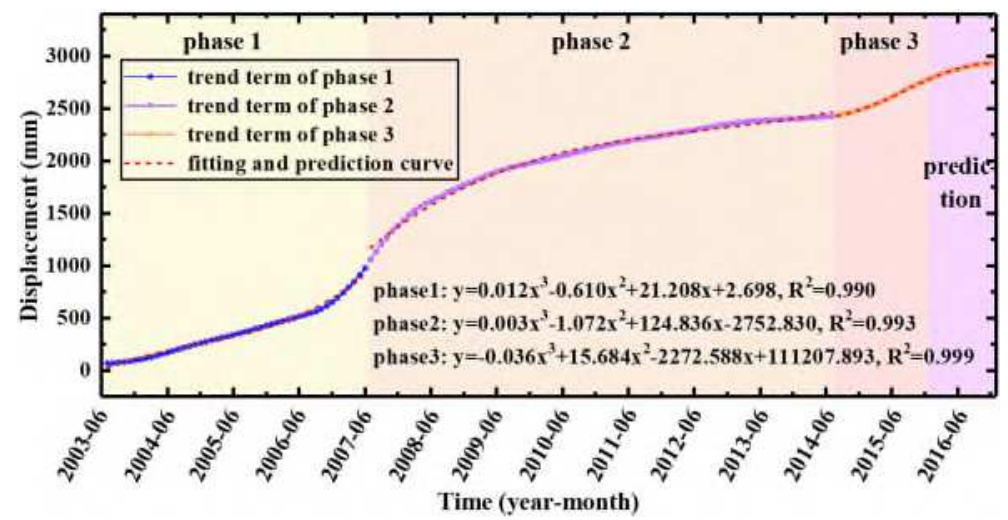

Fig. 9 Fitting and prediction curves of the trend term

Table 7 Parameters of the trend term of displacement based on polynomial fitting

\begin{tabular}{cccccccc}
\hline Phase & $\mathrm{a}$ & $\mathrm{b}$ & $\mathrm{c}$ & $\mathrm{d}$ & $\mathrm{R}^{2}$ & $\mathrm{MSE}$ & $\mathrm{RMSE}$ \\
\hline Phase 1 & 0.012 & -0.610 & 21.208 & 2.698 & 0.990 & 518.271 & 22.766 \\
Phase 2 & 0.003 & -1.072 & 124.836 & -2752.830 & 0.993 & 780.995 & 27.946 \\
Phase 3 & -0.036 & 15.684 & -2272.588 & 111207.893 & 0.999 & 20.876 & 4.569 \\
All training samples & $/$ & $/$ & $/$ & $/$ & 0.994 & 563.729 & 23.743 \\
Prediction samples & $/$ & $/$ & $/$ & $/$ & 0.991 & 16.510 & 4.063 \\
\hline
\end{tabular}

\section{Periodic and random term prediction}

The FOA-BPNN was used to train and predict the periodic and random terms of the ZG93 displacement. In the FOA algorithm, the population size $(p)$ was set as 10 , and the iterations of FOA $(k)$ was set to 1000. In the BPNN model, the hidden layer of the neural network was set to 12 based on multiple attempts. A total of six FOA-BPNN models were built, including individual periodic prediction models for V1, V2, and V3, and individual random prediction models for $\mathrm{V} 1, \mathrm{~V} 2$, and $\mathrm{V} 3$. Training and prediction results for the periodic and random terms are shown in Fig. 10.

It can be known that the FOA-BPNN models established in this paper have achieved good accuracy in the prediction of periodic and random displacement of the Baishuihe landslide. From the results of residual error analysis, in the training process of the model, the residual error of displacement was relatively stable, which also verified the robustness and reliability of the model. For the prediction samples, there were some fluctuations in the residual error. The prediction accuracy of the model is analyzed in Discussion section.

\section{Total displacement}

Prediction results of the total displacement of landslide can be obtained by summation the trend term, periodic term, and random term of displacement, as shown in Fig. 11. It can be known that the prediction model established in this paper has achieved good accuracy in the total displacement of monitoring point ZG93. Residual error results showed that in June 2007, there was a big difference between the total displacement training value and the actual value, resulting in the obvious mutation of the residual error. This was because in the three parts of landslide displacement (trend, periodic, random), the trend displacement accounts for more than 85\%. In June 2007, it was the boundary between Phase 1 and Phase 2, where there were some differences in the training results of the two polynomial fitting functions, resulting in a large residual error in the total displacement. For the prediction samples, the residual error was relatively stable. 


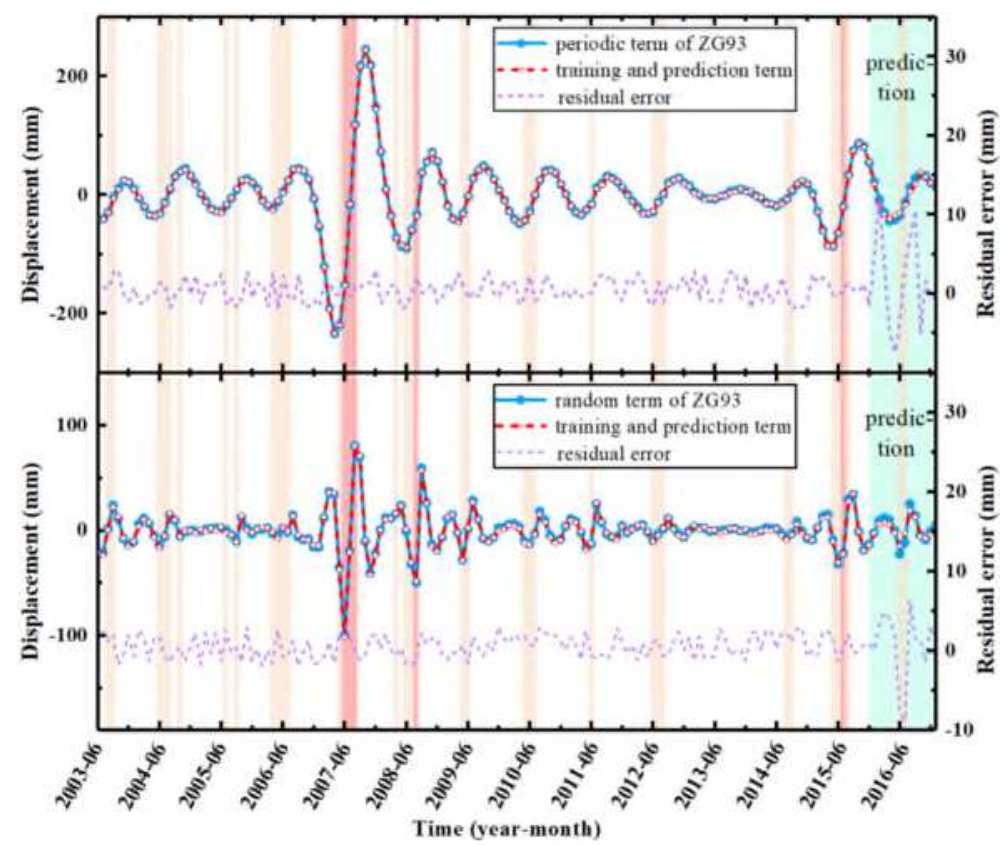

Fig. 10 Training and prediction curves of the periodic and random term

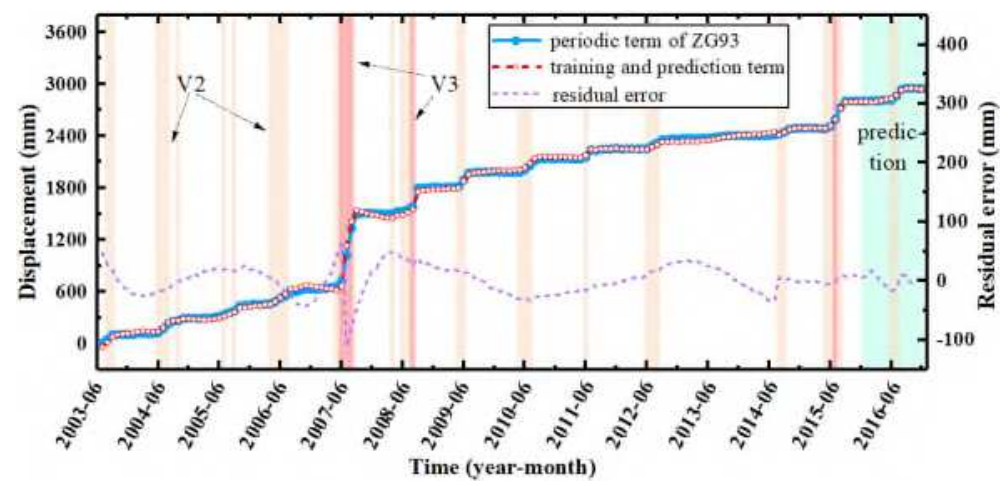

Fig. 11 Training and prediction curves of the total displacement

\section{Discussion}

When the time series analysis method is used to predict the landslide displacement, the trend displacement is relatively easy to be predicted. Therefore, choosing the appropriate periodic displacement prediction model is the key to improve the effect of landslide displacement prediction. The landslide prediction model has experienced rapid development in the past 50 years, and various machine learning models have been widely used in landslide displacement prediction. However, each algorithm has its limitations. For instance, SVM has low computational complexity, but it is sensitive to the choice of parameters and kernel function. Decision Tree model does not need any prior assumptions on the data, but the demand for sample size is relatively large, and the function of dealing with missing values is quite limited. ELM uses the principle of least square and pseudo inverse matrix to solve the problem, which is only suitable for single hidden layer neural network. BPNN has strong self-learning, self-adaptive ability, and good generalization ability, but it is prone to slow convergence. Therefore, it is of profound significance to select the appropriate optimization algorithm to optimize the machine model for the accuracy of displacement prediction of landslides. In this paper, based on the VMD and data mining results, the FOA-BPNN was used to predict the periodic and random term of monitoring point ZG93 displacement. BPNN, SVM, and ELM algorithms were chosen as the comparison models (Model 2 4). Performance of various 
displacement prediction models of the Baishuihe landslide are shown in Table 8 and Fig. 12. The prediction accuracy of the FOA-BPNN model was the highest. The $\mathrm{R}^{2}$ reached 0.977 , and its RMSE was only 10.041. In contrast, the proposed model can improve the accuracy of landslide displacement prediction..

Table 8 Performance of various displacement prediction models of the Baishuihe landslide

\begin{tabular}{ccccc}
\hline \multirow{2}{*}{ Model } & \multirow{2}{*}{ Algorithm's combination } & \multicolumn{3}{c}{ Prediction term } \\
\cline { 3 - 5 } & & $\mathrm{R}^{2}$ & MSE & RMSE \\
\hline Model 1 & VMD+TSC+Apriori+FOA-BPNN & 0.977 & 100.828 & 10.041 \\
Model 2 & VMD+TSC+Apriori+BPNN & 0.923 & 340.481 & 18.452 \\
Model 3 & VMD+TSC+Apriori+SVM & 0.944 & 282.566 & 16.81 \\
Model 4 & VMD+TSC+Apriori+ELM & 0.877 & 940.462 & 30.667 \\
\hline
\end{tabular}

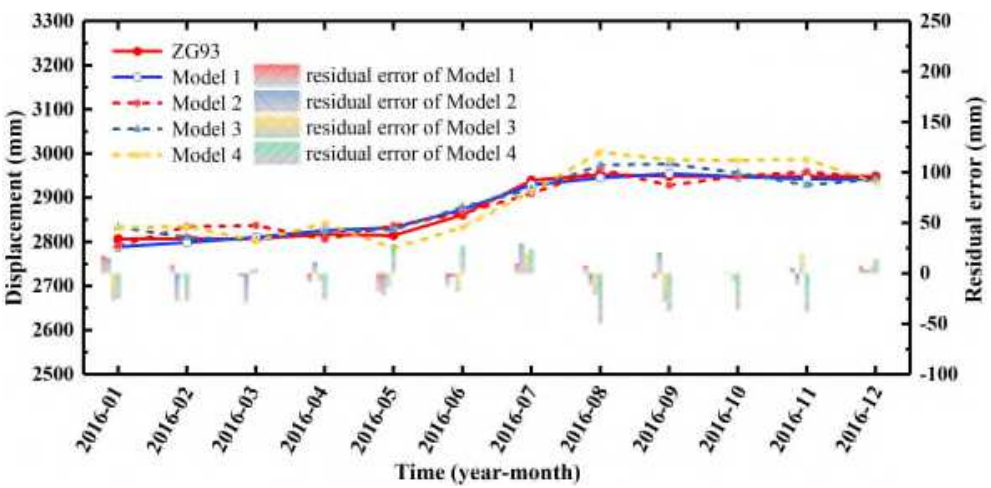

Fig. 12 Prediction curves of the total displacement

In this paper, ZG93 monitoring data was selected for displacement prediction, and the monitoring data of points ZG118 and XD01 were added to increase the sample size and overcome model overfitting error, as well as to provide a better representative prediction of the overall landslide displacement. The accuracy of various models for ZG93 displacement prediction has been discussed. The monitoring points of ZG118 and XD01 in 2016 were used for the model validation. Measured and prediction displacements of ZG93, ZG118, XD01 are shown in Fig. 13. ${ }^{2}$ between the measured and prediction displacements of ZG118 and XD01 were 0.977 and 0.978. RMSE of these two monitoring points were 12.40 and 16.04 respectively. It can be seen that the model proposed in this paper has achieved ideal results for the displacement prediction of different monitoring points of landslide, which has high practicability and application value in the study of landslide displacement prediction. However, it's worth noting that due to the small amount of displacement data in V3 state of monitoring point (Table 6), the prediction results of XD01 have obvious errors in July 2016. Therefore, in order to obtain more ideal prediction results, the monitoring data of various states should be supplemented as much as possible. 


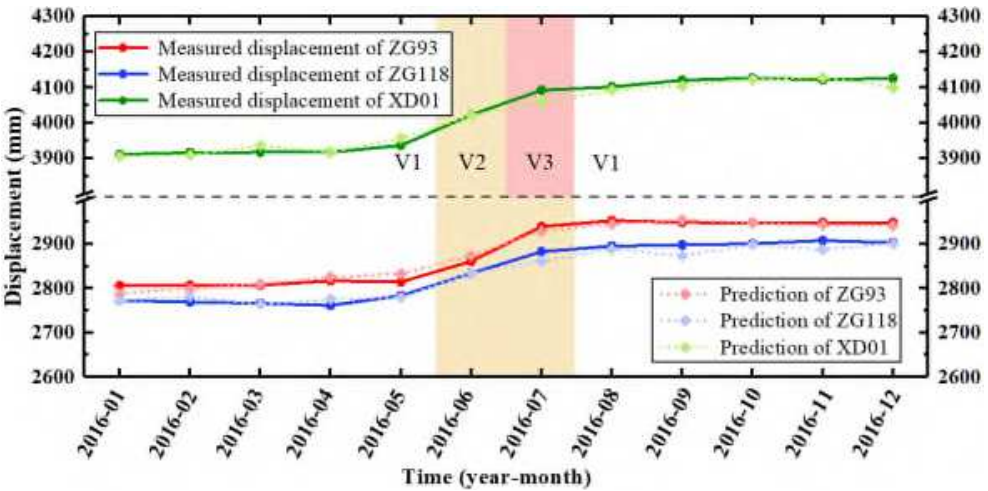

Fig. 13 Measured and prediction displacements of ZG93, ZG118, XD01

\section{Conclusions}

In this paper, the Baishuihe landslide, which has a typical "step-like" behavior, was taken as an example, and an integrated approach for landslide displacement prediction combined data mining and VMD-FOA-BPNN was proposed. The following results were obtained:

(1) Influenced by the fluctuation of reservoir water level and rainfall, the displacement curve of the Baishuihe landslide behaves "step-like". It has clear physical significance to decompose the landslide displacement into trend term, period term and random term by VMD, which can establish the relationship between the triggering factors and the displacement components.

(2) A total of 5447 association rules were generated based on the data mining. The association rules showed that the main factors controlling the V2 and V3 deformation of the landslide were the sharp fluctuation of reservoir water level and medium heavy rainfall.

(3) The trend term displacement was trained and predicted by one-dimensional cubic subsection function. And six FOA-BPNN models were used to train and predict the periodic and random term. The prediction model established in this paper has achieved good accuracy in the total displacement of monitoring point ZG93.

(4) An integrated approach for landslide displacement prediction including data mining was proposed. The prediction results showed that, compared with the current popular prediction model, this model can effectively improve the prediction accuracy, which has high practicability and application value in the study of landslide displacement prediction.

\section{Acknowledgments}

This research was supported by the National Natural Science Foundation of China (42007267, 41977244), the Fundamental Research Funds for the Central University, China University of Geosciences (Wuhan), and the National Key R\&D Program of China (2017YFC-1501301). The authors thank the colleagues in our laboratory for their constructive comments and assistance.

\section{References}

Agrawal, R., \& Srikant, R. (1994). Fast algorithms for mining association rules. In Proc. 20th int. conf. very large data bases, VLDB (Vol. 1215, pp. 487-499).

Chen, H., \& Zeng, Z. (2013). Deformation prediction of landslide based on improved back-propagation neural network. Cognitive computation, 5(1), 56-62.

Dragomiretskiy, K., \& Zosso, D. (2013). Variational mode decomposition. IEEE transactions on signal processing, 62(3), 531-544.

Dong, Y., Niu, J., Liu, Q., et al. (2021). A hybrid prediction model for wind speed using support vector machine and genetic programming in conjunction with error compensation. Stochastic Environmental Research and Risk Assessment, https://doi.org/10.1007/s00477-021-01996-0.

Fu, Z., Long, J., Chen, W., et al. (2021). Reliability of the prediction model for landslide displacement with step-like behavior. Stochastic Environmental Research and Risk Assessment, https://doi.org/10.1007/s00477-021-02029-6.

Goetz, J. N., Brenning, A., Petschko, H., et al. (2015). Evaluating machine learning and statistical prediction techniques for landslide susceptibility modeling. Computers \& geosciences, 81, 1-11. 
Guo, Z., Chen, L., Gui, L., et al. (2019). Landslide displacement prediction based on variational mode decomposition and WA-GWO-BP model. Landslides, 1-17.

Hong, H., Chen, W., Xu, C., et al. (2017). Rainfall-induced landslide susceptibility assessment at the Chongren area (China) using frequency ratio, certainty factor, and index of entropy. Geocarto international, 32(2), 139-154.

Hong, H., Pourghasemi, H., \& Pourtaghi, Z. (2016). Landslide susceptibility assessment in Lianhua County (China): a comparison between a random forest data mining technique and bivariate and multivariate statistical models. Geomorphology, 259, 105-118.

Huang, F., Huang, J., Jiang, S., et al. (2017). Landslide displacement prediction based on multivariate chaotic model and extreme learning machine. Engineering Geology, 218, 173-186.

Intrieri, E., Carlà, T., \& Gigli, G. (2019). Forecasting the time of failure of landslides at slope-scale: A literature review. Earth-science reviews.

Juang, C. H., Dijkstra, T., Wasowski, J., et al. (2019). Loess geohazards research in China: Advances and challenges for mega engineering projects. Engineering geology, 251, 1-10.

Lenti, L., \& Martino, S. (2012). The interaction of seismic waves with step-like slopes and its influence on landslide movements. Engineering Geology, 126, 19-36.

Li, H., Xu, Q., He, Y., et al. (2019). Modeling and predicting reservoir landslide displacement with deep belief network and EWMA control charts: a case study in Three Gorges Reservoir. Landslides, 1-15.

Li, L., Wu, Y., Miao, F. et al. (2021). A hybrid interval displacement forecasting model for reservoir colluvial landslides with step-like deformation characteristics considering dynamic switching of deformation states. Stoch Environ Res Risk Assess, 35:1089-1112.

Li, X. Z., \& Kong, J. M. (2014). Application of GA-SVM method with parameter optimization for landslide development prediction. Natural Hazards and Earth System Sciences, 14(3), 525.

Lian, C., Zeng, Z., Yao, W., et al. (2014). Ensemble of extreme learning machine for landslide displacement prediction based on time series analysis. Neural Computing and Applications, 24(1), 99-107.

Lian, C., Zeng, Z., Yao, W., et al. (2014). Extreme learning machine for the displacement prediction of landslide under rainfall and reservoir level. Stochastic environmental research and risk assessment, 28(8), 1957-1972.

Liu, Yong., Xu, Chang., Huang, Biao., et al. (2020). Landslide displacement prediction based on multi-source data fusion and sensitivity states. Engineering Geology, 271: 105608.

McClelland, J., Rumelhart, D., \& PDP Research Group. (1986). Parallel distributed processing. Explorations in the Microstructure of Cognition, 2, 216-271.

Miao, F., Wu, Y., Li, L., et al. (2018a). Centrifuge model test on the retrogressive landslide subjected to reservoir water level fluctuation. Engineering Geology, 245, 169-179.

Miao, F., Wu, Y., Xie, Y., et al. (2018b). Prediction of landslide displacement with step-like behavior based on multialgorithm optimization and a support vector regression model. Landslides, 15(3), 475-488.

Pan, W. T. (2012). A new fruit fly optimization algorithm: taking the financial distress model as an example. Knowledge-Based Systems, 26, 69-74.

Shihabudheen, K. V., Pillai, G. N., \& Peethambaran, B. (2017). Prediction of landslide displacement with controlling factors using extreme learning adaptive neuro-fuzzy inference system (ELANFIS). Applied Soft Computing, 61, 892-904.

Tang, H., Wasowski, J., \& Juang, C. H. (2019). Geohazards in the three Gorges Reservoir Area, China-Lessons learned from decades of research. Engineering Geology, 105267.

Wang, J., Di, Y., \& Rui, X. (2019). Research and application of machine learning method based on swarm intelligence optimization. Journal of Computational Methods in Sciences and Engineering, 19(S1), 179-187.

Wu, Y., Miao, F., Li, L., et al. (2017). Time-varying reliability analysis of Huangtupo Riverside No. 2 landslide in the Three Gorges Reservoir based on water-soil coupling. Engineering Geology, 226:267-276.

Xu, S., \& Niu, R. (2018). Displacement prediction of Baijiabao landslide based on empirical mode decomposition and long short-term memory neural network in Three Gorges area, China. Computers \& Geosciences, 111, 87-96.

Yang, B., Yin, K., Lacasse, S., et al. (2019). Time series analysis and long short-term memory neural network to predict landslide displacement. Landslides, 16(4), 677-694.

Zhou, C., Yin, K., Cao, Y., et al. (2016). Application of time series analysis and PSO-SVM model in predicting the Bazimen landslide in the Three Gorges Reservoir, China. Engineering geology, 204, 108-120.

Zhou, C., Yin, K., Cao, Y., et al. (2018). Displacement prediction of step-like landslide by applying a novel kernel extreme learning machine method. Landslides, 15(11), 2211-2225.

Zhang, Y., Tang, J., Liao, R., et al. (2021). Application of an enhanced bp neural network model with water cycle algorithm on landslide prediction. Stochastic Environmental Research and Risk Assessment, 35:1273-1291. 